\title{
Experimental study of the transitions between synchronous chaos and a periodic rotating wave
}

\author{
Esteban Sánchez \\ Area de Tecnología Electrónica, Universidad de Salamanca, E-37700 Béjar, Salamanca, Spain \\ Diego Pazóa) \\ Max-Planck-Institut für Physik komplexer Systeme, Nöthnitzer Straße 38, 01187 Dresden, Germany \\ Manuel A. Matías ${ }^{\text {b) }}$ \\ Instituto Mediterráneo de Estudios Avanzados, IMEDEA (CSIC-UIB), E-07122 Palma de Mallorca, Spain
}

(Received 7 April 2006; accepted 18 July 2006; published online 13 September 2006)

\begin{abstract}
In this work we characterize experimentally the transition between periodic rotating waves and synchronized chaos in a ring of unidirectionally coupled Lorenz oscillators by means of electronic circuits. The study is complemented by numerical and theoretical analysis, and the intermediate states and their transitions are identified. The route linking periodic behavior with synchronous chaos involves quasiperiodic behavior and a type of high-dimensional chaos known as chaotic rotating wave. The high-dimensional chaotic behavior is characterized, and is shown to be composed actually by three different behaviors. The experimental study confirms the robustness of this route. (C) 2006 American Institute of Physics. [DOI: 10.1063/1.2335815]
\end{abstract}

Building arrays of coupled oscillators is quite an obvious way of creating high-dimensional, albeit still finite, phase spaces. The most intensively studied behavior of this type of system is the case in which the rhythms of these oscillators become entrained or synchronized, which in the extreme case of complete synchronization implies that the dynamics of the coupled system takes place in a small subset of the phase space whose dimension corresponds to a single oscillator: the synchronization manifold. Nonetheless, it is clear that coupled oscillators are natural candidates to study more complicated behaviors, namely those in which the dynamics is high-dimensional and chaotic. In previous work a transition between chaotic synchronization and periodic rotating waves was described for an array of three Lorenz oscillators coupled unidirectionally in a ring. Here we shall further demonstrate the dynamical richness, and also the robustness of the route linking these states through an experimental study. Two symmetry-related quasiperiodic attractors will be shown to lead to a high-dimensional chaotic attractor, and, later, the transition from this attractor to synchronized chaos will be shown.

\section{INTRODUCTION}

In nonlinear dynamics many studies have helped to unveil the richness of transitions, including the transitions to chaotic behavior, by means of systems with a low phase space dimension (typically 3 for continuous systems and 1 or 2 for maps). On the other extreme, one has the case of continuous systems described by a nonlinear partial differential

\footnotetext{
${ }^{a)}$ Present address: Instituto de Física de Cantabria (CSIC-UC), E-39005 Santander, Spain.

${ }^{b)}$ Electronic mail: manuel@imedea.uib.es. URL: http://www.imedea.uib.es/ PhysDept/
}

equation, and that formally live in an infinite-dimensional phase space. In practice, many important complex systems like the atmosphere, the ocean or the brain are highdimensional objects. In order to understand them one usually resorts to some kind of statistical assumption (see, e.g., Ref. 1 ), whose validity increases as the number of active modes involved in the description. The goal of the present work is to study the intermediate range by focusing on a dynamical system of medium dimensionality applying the tools used routinely for low-dimensional systems.

A straightforward method to design high-dimensional systems is based on coupling together several lowdimensional systems (oscillators). In the present study we use as a unit element the Lorenz oscillator ${ }^{2}$ (arising itself from a drastic truncation of Navier-Stokes equations). Coupled oscillators have been much studied in the context of synchronization (both periodic ${ }^{3,4}$ and, since the 1980s, chaotic $^{5}$ ). Chaotic synchronization became a subject of interest for a wide community of scientists (from the viewpoint of both theory and applications) after the work by Pecora and Carroll in $1990 .^{6}$ Thus, a large number of studies have been devoted to chaotic synchronization and its applications (see, e.g., Refs. 7-9 and references therein). In our case it is of greater interest the regime in which complete synchronization is lost, as this will lead to a higher dimensional attractor. A widely studied class of these type of behaviors is the blowout bifurcation, leading to riddling behavior, ${ }^{8,10}$ as are several types of intermittent behavior, like on-off intermittency. Of interest is that one can classify types of attractors obtained after these transitions, the so-called chaotic hierarchies. ${ }^{11}$

Interestingly enough, loss of (complete) synchronization of coupled chaotic oscillators leads quite often to phase relations among the coupled oscillators. The selection of these phase patterns is a topic of large interest in a biological con- 
text, with applications ranging from neuroscience and brain function ${ }^{12}$ to animal locomotion. ${ }^{13}$ In the latter case, circular geometries of coupled cells are used for modeling central pattern generators ${ }^{14}$ because the system's symmetries provide the different phase patterns observed for different gaits.

Some previous studies have focused on the emergence of certain phase patterns from synchronized states, mostly periodic ${ }^{15-17}$ oscillators. A class of phase patterns in chaotic dynamics occur after a symmetric Hopf bifurcation on a chaotic synchronized state takes place. ${ }^{18}$ In particular, a chaotic rotating wave (CRW) appears, as predicted by symmetry arguments. ${ }^{14}$ In the present work we shall try to shed light on the transitions among the different spatio-temporal patterns occurring in this kind of system, in the spirit of previous works, ${ }^{19,20}$ not only in a neighborhood of a synchronized state. Some of these spatio-temporal structures are in fact coherent structures, such as the periodic, ${ }^{18,21,22}$ and chaotic ${ }^{23}$ rotating waves, PRW and CRW, respectively, that have been found in rings of coupled chaotic oscillators, and then also studied by other authors, ${ }^{24-26}$ including the transition between different modes. ${ }^{27}$ Other transitions between periodic and chaotic waves are reported in Ref. 28. The present work offers an experimental survey of the states between synchronous chaos and a periodic rotating waves, including three types of chaotic rotating wave and quasiperiodicity.

Our approach to this problem has been both numerical and experimental (our experimental setup-consisting of electronic circuits - is described in Sec. III). Digital and analog simulation are quite complementary, and their combined use allows a deeper understanding of problems that may be too difficult if studied with theory and digital simulation alone. The might of analog simulation may be summarized in the following facts: ${ }^{29}$ (i) it readily enables us to survey large volumes in parameter space looking for interesting phenomena-guiding and optimizing numerical computations; (ii) analog circuits are real physical systems, and so its phenomenology is genuine, and the observed behaviors are robust against parameter mismatch and in the presence of noise; (iii) one can play with time scales to make dynamics slower or faster at convenience. In particular, in the case that one wishes to study a small region in parameter space, as is the case of the quasiperiodic dynamics studied in this work (cf. Sec. IV B), through analog simulation it is possible to locate-and check the robustness of - the desired behaviors in quite a fast and convenient way (to be compared with performing systematic searches using fine meshes in parameter space).

An important problem of recent interest is shadowability, that is the possibility of modeling in the computer certain types of nonlinear deterministic dynamical systems. In particular, in recent theoretical studies it has been advocated that some of these systems cannot be properly modeled, ${ }^{30}$ i.e., are not shadowable, to the point that in these systems working with experimentally measured time series makes more sense than working with a mathematical model. One of the situations in which this may happen is when one (or more) Lyapunov exponents fluctuate around zero, a manifestation of nonhyperbolicity. Coupled chaotic oscillators are strong candidates to exhibit this behavior. Our system is a candidate to exhibit this form of nonhyperbolicity, while other mechanisms like tangencies between stable and unstable manifolds cannot be excluded either.

Nonhyperbolic systems, as the Lorenz oscillators that makes part of our system, may also be sensitive to noise, ${ }^{31}$ including the unavoidable noise always present in experiments. For this reason, it makes a lot of sense to try to double check the predictions of numerical simulation, specially regarding such strongly sensitive behaviors as quasiperiodic dynamics and high-dimensional chaotic attractors, as these attractors are very fragile.

The paper is organized as follows: Section II presents a short description of the system, and an overview of the different behaviors in parameter space by means of a numerical computation of the Lyapunov exponents. In Sec. III, the electronic setup and some of the techniques used in this paper are explained. Section IV gives rise to the experimental results, that are analyzed by means of fast Fourier transforms (FFTs) and Poincaré cross sections. Finally, in Sec. V the main results contained in this paper are discussed.

\section{BACKGROUND AND SYSTEM OVERVIEW}

In this section we introduce the model and present a numerical exploration of some of basic features of the system.

\section{A. The system}

Our system consists of a ring built with three Lorenz oscillators coupled unidirectionally using the partial replacement method. ${ }^{32}$ In this situation, the differential equations governing the dynamics read:

$$
\dot{x}_{i}=\sigma\left(y_{i}-x_{i}\right), \quad \dot{y}_{i}=R \underline{x_{i}}-y_{i}-x_{i} z_{i}, \quad \dot{z}_{i}=x_{i} y_{i}-b z_{i},
$$

where $x_{i}=x_{i-1}$ if $i \neq 1$ and $x_{1}=x_{3}$. This coupling method makes possible the chaotic synchronization of the three cells for some values of the parameters $R, \sigma$, and $b$. A physical interpretation can be found for this kind of coupling, apart from being a generalization of Pecora-Carroll ${ }^{6}$ master-slave coupling. Asymmetry in a coupling models advection (i.e., transport in one direction) in a discretely coupled system. In particular, the simple unidirectional (gradient) coupling, $K\left(x_{i-1}-x_{i}\right)$, represents the completely asymmetric case with only advection (and no diffusion). Our coupling, Eq. (1), represents the case in which the coupling constant, $K$, is made exactly equal to the $R$ parameter. This has the advantage in the electronic implementation of not introducing more parameters to the system, which makes it easier to calibrate and manipulate the setup, in addition to having a straightforward implementation (cf. Sec. III).

In previous studies ${ }^{18,19,21,33}$ some interesting phenomena had been unveiled for this system, and here we put all these behaviors in a unified framework.

A useful representation of Eq. (1) can be written in terms of the discrete Fourier modes of the system, that are defined in the following way, as a function of the oscillators coordinates: 


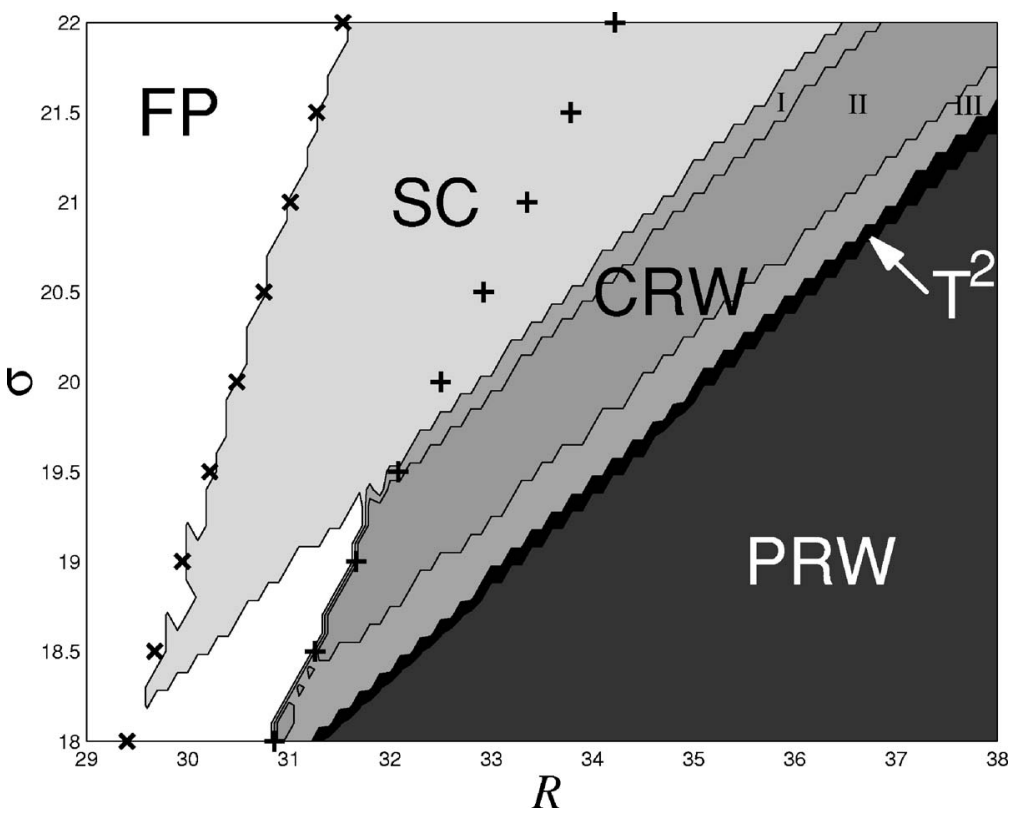

FIG. 1. Regions of the plane $(R, \sigma)$, for $b=3$, where different states are numerically found for a system composed of three Lorenz oscillators coupled according to Eq. (1). Notation: FP, fixed point; SC, synchronous chaos; CRW, chaotic rotating wave (subregion II exhibits a second LE above zero, i.e., hyperchaos, we took $10^{-3}$ as a cutoff to consider $\lambda_{2}$ positive); $T^{2}$, twofrequency quasiperiodicity; PRW, periodic rotating wave. The symbols " $\times$ " and "+" indicate the loci of $R_{1}$ and $R_{H}$ (see main text), respectively.

$$
X_{k}=\frac{1}{3} \sum_{j=1}^{3} x_{j} \exp \left[\frac{2 \pi i(j-1) k}{3}\right], \quad k=0,1
$$

with analogous expressions for $Y_{k}$ and $Z_{k}$. Note that the mode $k=0$ is nothing but a simple average along the ring. Also it must be pointed out that working with the Fourier modes $\left(X_{k}, Y_{k}, Z_{k}\right)$ is equivalent to working with the oscillators coordinates $\left(x_{j}, y_{j}, z_{j}\right)$. In fact, it is just a change from nine real variables to three real and three complex variables, and therefore no information is lost.

It is a general principle that, when working with assemblies of identical oscillators, desynchronization occurs associated with the instability of some discrete mode of the system. ${ }^{10,34}$ In this way (complete or identical) chaotic synchronization is understood as a state where all transverse modes $(k>0)$ are identically zero and asymptotically stable. ${ }^{35}$ Also, the mode that determines the motion into the synchronization manifold $(k=0)$ exhibits chaotic dynamics. This means that each oscillator follows exactly the same chaotic trajectory, in our case inside the Lorenz chaotic attractor. Due to the small size of our ring there is only one complex transverse mode $k=1$.

In the remainder of this section, we first introduce the different spatio-temporal structures that appear depending on the parameters of the system. These structures correspond to different behaviors and are identified by means of numerical computations. Then, we present a brief qualitative explanation of the general behavior and a detailed study of a route that links these structures.

\section{B. Lyapunov exponents and attractors}

In previous studies, ${ }^{23}$ it was demonstrated that the computation of the transverse Lyapunov exponents to the synchronization manifold allows us to determine (approximately) the region of the parameter space where synchronized chaos is stable. Nonetheless, if one wishes to characterize better the spatio-temporal structures emerging from desynchronization, it is worthwhile to compute the spectrum of Lyapunov exponents (LEs) for the whole set of variables (nine in our case). Since the LEs measure the exponential rate of growth along different directions in an attractor, they can be used as an identification tool of qualitative changes in the dynamics of the attractor.

The method used for the calculation has been the one developed by Benettin et al. ${ }^{36}$ and Shimada and Nagashima ${ }^{37}$ and described in Ref. 38. For the orthonormalization process we have used a modified Gram-Schmidt method. The integration of the system of differential equations (1) and the copies of the linearized system has been done by means of an adaptive step size algorithm based on a fourth order RungeKutta method. ${ }^{39}$ A first characterization of the different behaviors of the system has been achieved by keeping constant one parameter, $b=3$, and computing the four largest Lyapunov exponents (LEs) as a function of the parameters $\sigma$ and $R$.

The results are presented in Fig. 1, and were obtained by computing trajectories of $8 \times 10^{4}$ t.u., after transients of $10^{5}$ t.u. Then, using as a criterion the Lyapunov spectrum, the different attractors have been characterized on the $(R, \sigma)$ plane (see Table I for the correspondence between the Lyapunov spectrum and the corresponding attractor). Thus, we find that, in the interval $R \in[29,38]$, there is a

TABLE I. Correspondence between Lyapunov spectra and attractors. Fourth to ninth Lyapunov exponents are negative in all cases.

\begin{tabular}{ll}
\hline \hline Lyapunov spectra & Attractor \\
\hline$(-,-,-,-, \cdots)$ & Fixed point \\
$(+, 0,-,-, \cdots)$ & Synchronous chaos \\
$(+, 0,0,-, \cdots)$ & Chaotic rotating wave I and III \\
$(+,+, 0,-, \cdots)$ & Chaotic rotating wave II \\
$(0,0,0,-, \cdots)$ & 3-Torus \\
$(0,0,-,-, \cdots)$ & 2-Torus \\
$(0,-,-,-, \cdots)$ & Periodic rotating wave \\
\hline \hline
\end{tabular}


region when $R$ is small (denoted as FP in Fig. 1) where all the LEs are negative, which indicates that the three oscillators "collapse" to the same fixed point $C_{ \pm}$ $=[ \pm \sqrt{b(R-1)}, \pm \sqrt{b(R-1)}, R-1]$ (the meaning of the symbols " $\times$ " and "+" is explained below in this subsection). Also, there exists a region with synchronous chaos (SC) where the Lyapunov spectrum contains one positive and one null exponents.

For larger values of the parameter $R$, the synchronized state becomes unstable through a soft "blowout" (nonhysteretic) bifurcation. This means that, as $R$ is increased, more and more unstable periodic orbits (UPOs) embedded into the synchronous attractor become transversely unstable through Hopf bifurcations, in such a way that above a particular value of $R$ (depending on $\sigma$ ) the synchronized state becomes unstable on average (blowout bifurcation). ${ }^{40}$ This bifurcation is preceded by the phenomenon of attractor bubbling, ${ }^{41}$ that occurs when the system starts visiting transversely unstable UPOs which makes the attractor size increase. The transition may be classified as soft, because the trajectory does not depart significantly from the (unstable) synchronous state, and this indicates that instabilities of the UPOs occur through supercritical Hopf bifurcations. This oscillatory instability gives rise to an interesting spatio-temporal structure called chaotic rotating wave (CRW).

CRWs arise in systems exhibiting a Hopf bifurcation not on a steady state, as it is customary, but on a chaotic state. The Hopf bifurcation takes place in the $k=1$ complex mode (transverse subspace), with fixed point dynamics at the synchronized state, leaving the $k=0$ chaotic synchronization manifold qualitatively unchanged. Thus, close to the onset of the bifurcation, region I, a CRW is the combined dynamics stemming from a chaotic dynamics in the synchronization manifold with the oscillation introduced by the Hopf bifurcation, that is symmetric ${ }^{14}$ as it stems from the $k=1$ mode, that is present in the system due to coupling.

The CRW state in region I was first found for a ring of Chua's circuits ${ }^{23}$ and later for a ring of Lorenz oscillators. ${ }^{19}$ An analogous behavior was found in Ref. 25 for coupled maps in a ring. For this system, the CRW is characterized by a fast oscillation (compared to the time scale of the chaotic oscillation), introduced by the Hopf bifurcation. The CRW is associated with a phase difference of $2 \pi / n$ between neighboring oscillators (where $n$ is the number of units in the ring), superimposed to the chaotic motion. We distinguish three regions with this state depending on the LEs. In regions I and III, there exists one positive and two vanishing Lyapunov exponents (the degeneracy of this exponent is not theoretically proved, so it is plausible that one exponent has very small magnitude although it is indistinguishable from zero in practice). In region II, instead, there are two (clearly) positive and one vanishing LEs.

At larger values of $R$ we observe a small region that exhibits two-frequency quasiperiodicity $\left(T^{2}\right)$. The Lyapunov spectrum does not contain any positive exponent, hence chaos has disappeared, instead there are two null Lyapunov exponents which correspond to the two incommensurate frequencies of the quasiperiodic regime. Finally, for large $R$ we find periodic dynamics (PRW) and, accordingly, only one Lyapunov exponent is zero, whereas the rest are negative.

It is interesting to notice that, in Fig. 1, there exists a wedge-shaped region where a fixed point state replaces the CRW dynamics. This is due to the fact that inside the stripeshaped region of the $(R, \sigma)$ plane bounded by the $\times$ and + symbols, the Lorenz system exhibits tristability: two fixed points $\left(C_{ \pm}\right)$and the chaotic attractor. The fixed points become unstable, though a subcritical Hopf bifurcation, at

$$
R_{H}=\frac{\sigma(\sigma+b+3)}{\sigma-b-1},
$$

whereas, as explained by Yorke and Yorke, ${ }^{42}$ the chaotic attractor is born from the unstable chaotic set at a boundary crisis at some $R_{1}<R_{H}$ ( $\sigma$ fixed). The value of $R_{1}$ cannot be found analytically, but it is easy to obtain it numerically, because at this value there exists a double heteroclinic connection between the fixed point at the origin and each unstable cycle surrounding $C_{+}$and $C_{-}$.

The loci of $R_{1}$ and $R_{H}$ on the $(R, \sigma)$ plane are depicted in Fig. 1 with " $\times$ " and "+" symbols, respectively. For the Lorenz system, the basin of attraction of the chaotic attractor is much larger than the basins of $C_{ \pm}$. For this reason, in the region bounded by the " $X$ " and "+" symbols and the symmetric Hopf bifurcation (the diagonal line that separates the SC and type I regions for $\sigma>19.25$ ) the synchronized chaos (SC) state is typically found, instead of fixed point behavior. Synchronized dynamics occurs inside the invariant synchronization manifold, and the chaotic state is reached for most initial conditions. However, when the SC becomes transversely unstable through the above mentioned symmetric Hopf bifurcation that should lead to a CRW, the existence of tristability strongly affects the dynamics. The potential CRW becomes only a transient state beyond the instability of the SC (plausibly the attractor touches its basin boundary). This explains the white, FP, wedge-like region for $\sigma<19.25$ and $R<32$. This wedge terminates at the line of "+" symbols as the Lorenz system is no longer tristable for $R>R_{H}$ (the chaotic attractor is then the only attractor of the Lorenz system).

\section{Transitions along the line $\sigma=20$}

Once the different behaviors have been identified, our goal is to perform a more detailed characterization of the attractors that appear in the system, and in their transitions. To make feasible the study we have varied $R$ while the other two parameters have been kept fixed: $\sigma=20$ and $b=3$. Figure 2(a) shows the values of the four largest Lyapunov exponents along a line for a slightly larger range of $R$ than in Fig. 1 .

In the right-most range of Fig. 2(a) the motion of every oscillator is periodic in such a way that adjacent oscillators exhibit a phase difference of $2 \pi / 3$. This state is what we denominate a periodic rotating wave (PRW) and is characterized by a fast frequency. At $R \approx 39.25$ this state undergoes a pitchfork bifurcation giving rise to two symmetry related PRWs. This transition occurs at the point where the second Lyapunov exponent touches zero.

Figure 2(b) shows that, when $R$ decreases, a couple of Lyapunov exponents reaches zero at $R \approx 35.26$, inducing a 

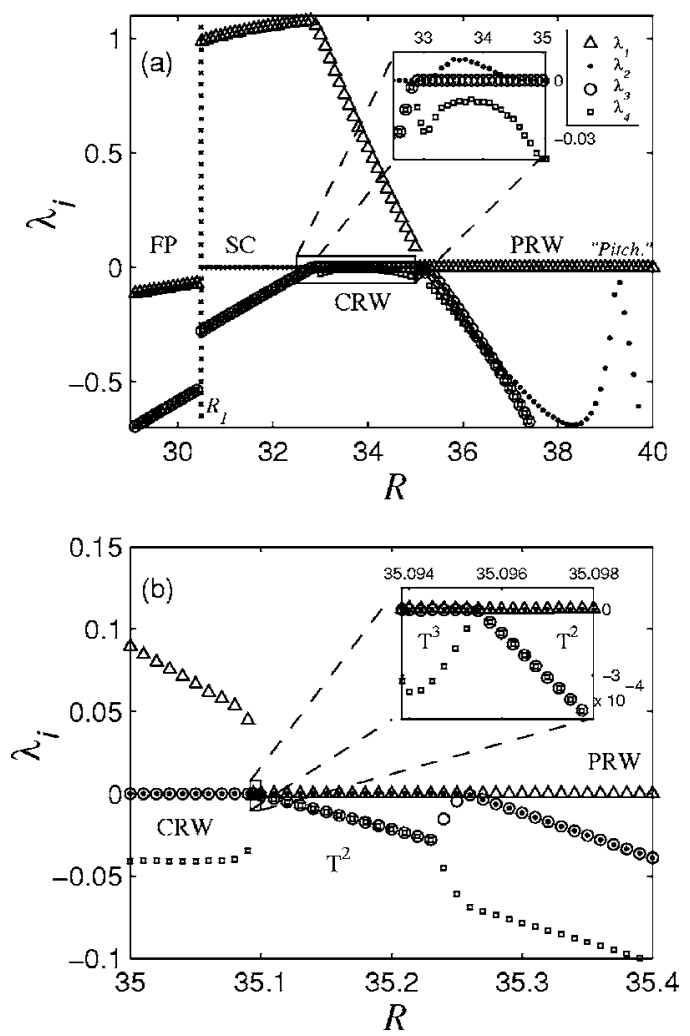

FIG. 2. (a) The four largest Lyapunov exponents of a ring of three unidirectionally coupled Lorenz systems, Eq. (1), along the line $\sigma=20$ (see Fig. 1). The inset shows the LEs from second to fourth in the interval with CRW dynamics. It may be seen that the second LE becomes slightly positive which indicates the existence of hyperchaos. (b) Detailed figure of the transition from PRW to CRW; the existence of three-frequency quasiperiodicity $T^{3}$ is confirmed in the inset where three vanishing LEs exist. The Lyapunov exponents have been obtained from the model Eq. (1).

Hopf bifurcation leading to quasiperiodicity (in accordance with Fig. 1). The new frequency does not exhibit any spatial dependence and manifests uniformly along the ring, being slower than the previous one. Also, it was demonstrated in a previous numerical work ${ }^{33}$ that an additional Hopf bifurcation leads to three-frequency quasiperiodicity, $T^{3}$, that exists in a very small interval of the parameter $R$ (it is not large enough to become visible in Fig. 1). It can be seen in the inset of Fig. 2(b) that a couple of Lyapunov exponents reaches zero at $R \approx 35.0955$ which precedes three-frequency quasiperiodicity $\mathbb{T}^{3}$ at lower values of $R$.

At the left of the quasiperiodic domain, $R<35.09384$, the CRW domain is found. It may be seen in the left most part of Fig. 2(b) and the inset of Fig. 2(a) that the zone of CRW with the largest values of $R$ exhibits two vanishing LEs (at least, very approximately). This region with degenerate zero LE is what we call region III. Degeneracy also occurs in region I. The dynamics in region III roughly resembles an intermittent motion between the two former mirror $\mathbb{T}^{3}$. In contrast, region I is characterized by a uniform chaotic motion (the average of the three oscillators that follows a trajectory very similar to that of a single Lorenz oscillator) plus an oscillation with the known phase shift of $2 \pi / 3$. Region II shows intermittent jumps between the dynamics of regions I and III. The behavior in region II, namely the transition from two zero Lyapunov exponents to a clearly positive and a zero Lyapunov exponent is similar to the one reported in Ref. 43 for two coupled Lorenz oscillators.

At this point, once we have a general view of the system, it is when we resort to the experiment to get more information about these behaviors.

\section{EXPERIMENTAL SETUP}

In the work presented here the experiment is not only a validation of the numerical results. Rather, both numerical and experimental studies were developed in parallel and both have interacted in a constructive way to achieve a more clear view of the behavior of the system. The experimental setup consists of a ring of three Lorenz analog circuits, representing Eq. (1). The electronic circuit consists of three integrators, one for each variable, and the nonlinear terms are represented using analog multipliers. The first step in designing the circuit is to rescale both the three state variables $x, y$, and $z$ in order to fit within the range $[-10 \mathrm{~V}, 10 \mathrm{~V}]$ (a more restricted range than that corresponding to the power supply, $[-15 \mathrm{~V}, 15 \mathrm{~V}])$, and such that the circuit operates in the frequency range of around $1 \mathrm{kHz}$. Thus, the circuit is basically the one described in Refs. 21 and 44, except that the time scaled has been slowed by an order of magnitude, for reasons explained below. The transformation applied to the variables of circuit $i$ is, thus, the following:

$\mathrm{x}_{i}=x_{i} / 5, \quad \mathrm{y}_{i}=y_{i} / 5, \quad \mathrm{z}_{i}=z_{i} / 10, \quad \mathrm{t}=t / A, \quad A=10^{2}$.

This rescaling of variables leads to the following set of differential equations, in which the variables, $\mathbf{x}_{i}, \mathbf{y}_{i}, \mathbf{z}_{i}$, are voltages across the three capacitors of circuit $i$, and in which the time is expressed in seconds,

$$
\begin{aligned}
& \dot{\mathrm{x}}_{i}=A \sigma\left(\mathrm{y}_{i}-\mathrm{x}_{i}\right), \quad \dot{\mathrm{y}}_{i}=A\left(R \underline{\mathrm{x}_{i}}-\mathrm{y}_{i}-10 \mathrm{x}_{i} \mathrm{z}_{i}\right), \\
& \dot{\mathrm{z}}_{i}=A\left(2.5 \mathrm{x}_{i} \mathrm{y}_{i}-b \mathrm{z}_{i}\right) .
\end{aligned}
$$

In Eq. (5), $\dot{\mathrm{x}}_{i}=d \mathrm{x}_{i} / d \mathrm{t}, \dot{\mathrm{y}}_{i}=d \mathrm{y}_{i} / d \mathrm{t}$, and $\dot{\mathrm{z}}_{i}=d \mathrm{z}_{i} / d \mathrm{t}$, as the time is expressed in rescaled units, and $\underline{x_{i}}$ has the same meaning as explained after Eq. (1).

The results have been obtained by sampling with a National Instruments NI-4454 acquisition board, that allows a maximum sampling rate of $51.2 \mathrm{kSa} / \mathrm{s}$ (or $\mathrm{kHz}$ ) simultaneously in its four available channels with a resolution of 16 bits. The possibility of sampling simultaneously the three circuits used in our study is a very important feature in the present study, as it allows a much better resolution of the nine-dimensional phase space that describes the system.

The reason why the circuits have been slowed down from the original implementation in Ref. 21 is to improve the accuracy in the determination of the Poincaré sections presented in Sec. IV. Poincaré sections have been obtained from interpolation of the digitally acquired data, and as the sampling frequency is fixed, and the circuits have been slowed down to minimize the error.

The spectra presented in Sec. IV consist of the power spectral density (PSD) of signal $\mathrm{x}_{1}(\mathrm{t})$ for different parameter values. In these calculations a sampling rate of $25.6 \mathrm{kSa} / \mathrm{s}$ has been used, and up to $2^{21}=2097152$ points have been 

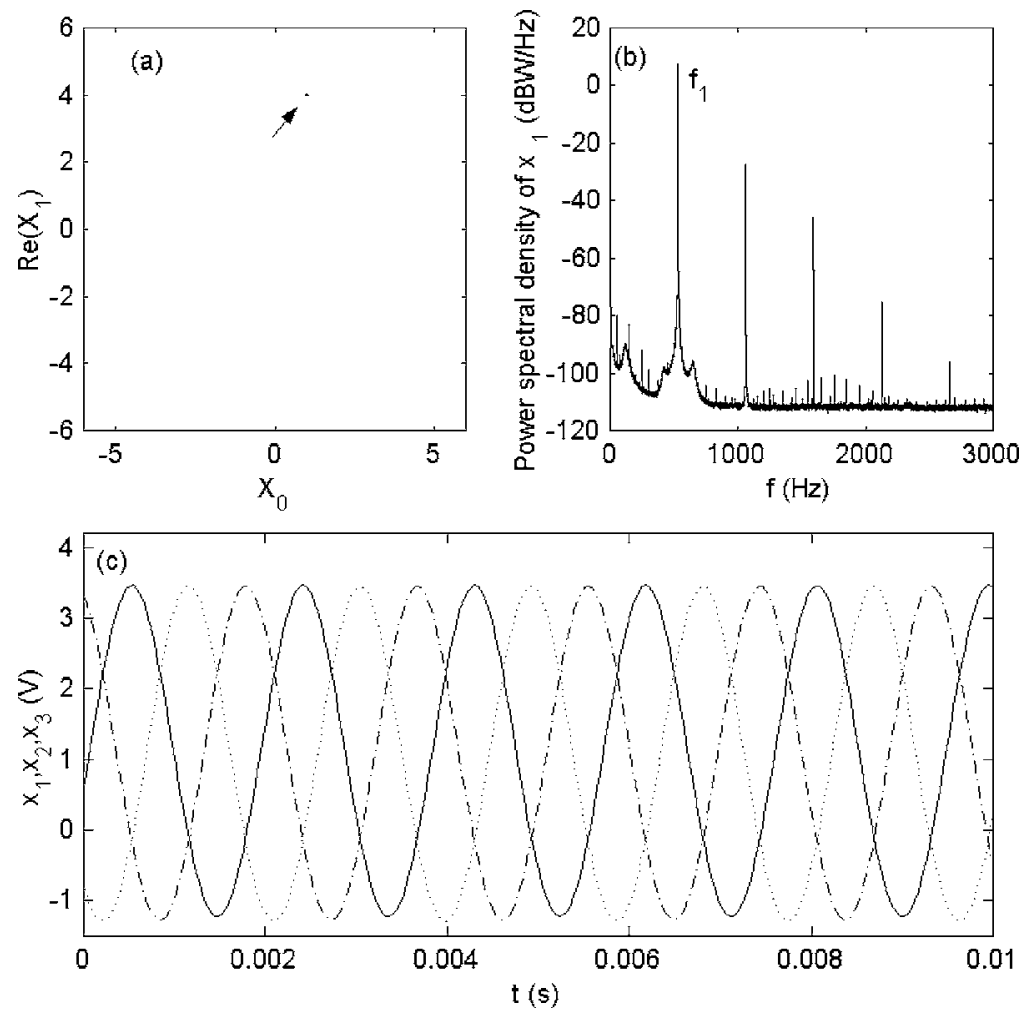

FIG. 3. Periodic motion (PRW): experimental results. (a) Poincaré surface of section for the system showing a point. The representation has been carried out using the complex mode representation of the system Eq. (2), where the axes correspond to the $\mathrm{X}$ variable, uniform mode $(k=0)$ and real part of the $k=1$ mode. The Poincaré section has been defined by the condition: $\operatorname{Re}\left(Z_{1}\right)=1$ with $\operatorname{Re}\left(\dot{Z}_{1}\right)>0$. (b) Power spectrum of variable $\mathrm{x}_{1}(\mathrm{t})$ showing the main peak and harmonics. (c) Temporal series of variable $\mathbf{x}$ in the three circuits showing a $2 \pi / 3$ phase shift between them. acquired in the cases of three-frequency quasiperiodicity and CRW dynamics to study the low frequency component of these behaviors.

\section{EXPERIMENTAL RESULTS}

In this section, a survey of the different behaviors obtained in our experimental setup is presented (for decreasing values of $R$ ). For each behavior we show a temporal evolution of the $\mathrm{X}$ variable (in one or all the oscillators), the power spectral density of the $\mathrm{x}_{1}$ coordinate, and a Poincare section defined, in all but one case, by the following condition:

$$
\mathrm{Z}_{0} \equiv \frac{1}{3} \sum_{i=1}^{3} \mathrm{z}_{i}=(R-1) / 10 \quad\left(\dot{\mathrm{Z}}_{0}>0\right)
$$

corresponding to the rescaled variable; for the original variables one would have $R-1$ on the right-hand side.

\section{A. Periodic rotating wave}

The state denominated periodic rotating wave (PRW) is found for large values of $R^{21,22}$ This state is characterized by a fast periodic motion of the oscillators of the array, and phase differences of $2 \pi / 3$ between adjacent oscillators. It can be considered as a motion where only the complex mode $k=1$ is active, whereas the $k=0$ (synchronous) mode is approximately at rest. If the ring were larger other modes could manifest. ${ }^{45}$

It was shown in a previous paper ${ }^{19}$ and explained above, that although for large $R$ there exists a centered PRW (whose dynamics may be accurately described in analytic form ${ }^{46}$ ), when $R$ is decreased this state undergoes a pitchfork bifurcation and two symmetry related PRWs appear. The temporal evolution of the oscillators of the array is shown in Fig. 3(c), and the $2 \pi / 3$ phase differences among them can be clearly appreciated. Also, in Figs. 3(a) and 3(b), we show the Poincaré section [different from that defined in Eq. (6) due to the absence of appreciable motion for the $k=0$ mode] and the power spectrum. For the Poincaré section we obtain, very approximately, a single point, as expected for a limit cycle. On the other hand, the power spectrum shows a peak at $f_{1}$ $=532.4 \mathrm{~Hz}$ and some harmonics.

\section{B. Quasiperiodic attractors}

A further decrease of $R$ leads to a supercritical NeimarkSacker bifurcation of the symmetry-related PRWs. The former limit cycles are substituted by quasiperiodic motions on the surface of two (mirror) tori $\left(\mathrm{T}^{2}\right)$. In Fig. 4 this new state is presented. The time scale of the new frequency $f_{2}$ is approximately on the same order of an isolated Lorenz oscillator and, opposed to the former frequency $f_{1}$, it has no spatial nature, i.e., it does no induce any phase shift between the oscillators [see Fig. 4(c)]. It can be seen in Fig. 4(a) that the Poincare section of the $\mathbb{T}^{2}$ is (quite accurately) a smooth closed curve. The power spectrum, shown in Fig. 4(b), exhibits two incommensurate frequencies $\left(f_{1}, f_{2}\right)$ as well as linear combinations of them $\left(f_{1}-f_{2}, f_{1}+f_{2}, f_{1}+2 \cdot f_{2}, \ldots\right)$ and harmonics $\left(2 \cdot f_{2}, 2 \cdot f_{1}, \ldots\right)$.

In Sec. II we noticed that numerical simulations indicated the existence of three-frequency quasiperiodic dynamics (see also Ref. 33 for a thorough study of threedimensional quasiperiodic dynamics for this system). Nonetheless, years ago, it was stated by Newhouse, Ruelle and Takens ${ }^{4}$ that small perturbations to the flow may convert three-frequency quasiperiodicity to a chaotic flow on a strange attractor lying in the 3 -torus $\left(T^{3}\right)$. Hence, the exis- 

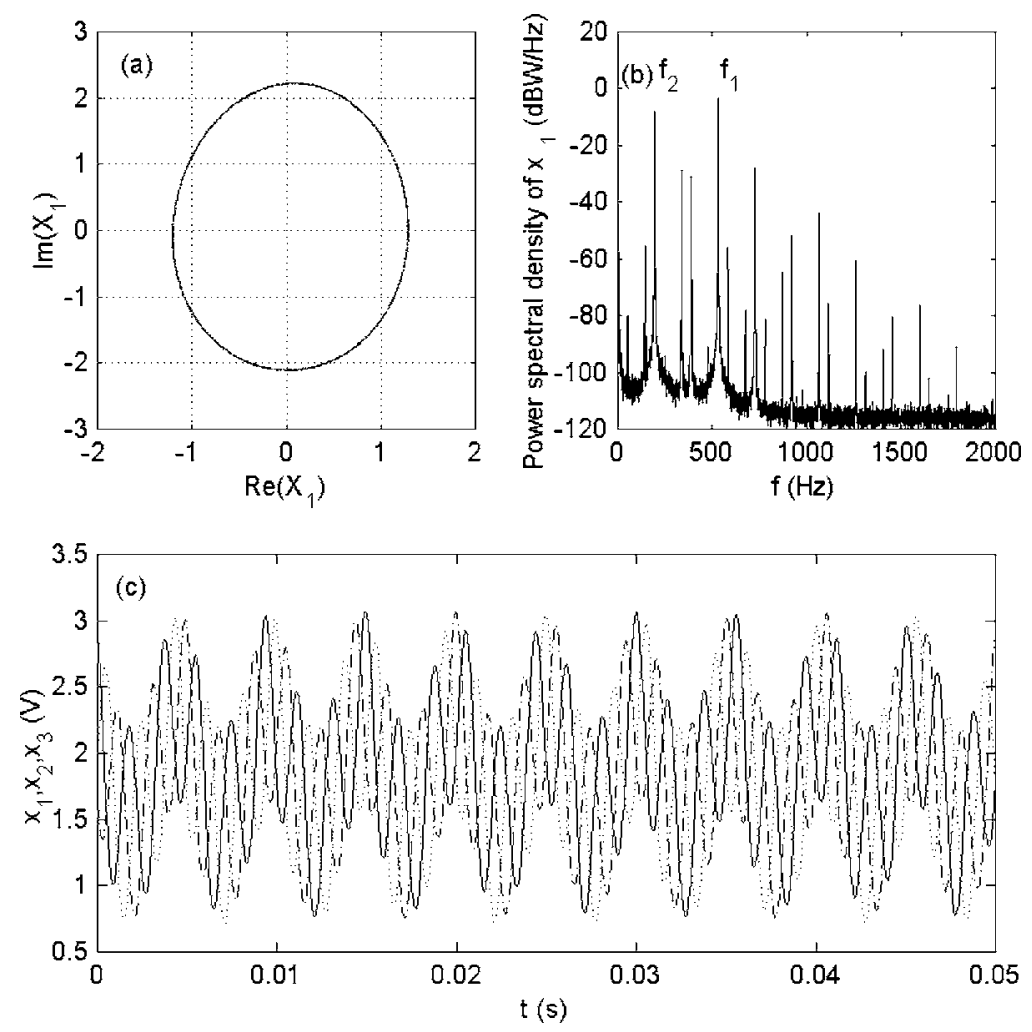

FIG. 4. Two-frequency quasiperiodic motion: experimental results. (a) Poincaré section for the system showing a closed line. The representation has been carried out using the complex mode representation of the system (see the caption of Fig. 3 for an explanation), where the axes correspond to the $\mathrm{x}$ variable, real and imaginary parts of the $k=1$ mode. The Poincaré section has been defined by Eq. (6). (b) Power spectrum of variable $\mathrm{x}_{1}(\mathrm{t})$ showing the two incommensurate frequencies and linear combinations of them. (c) Temporal series of variable $x$ in the three circuits showing the two oscillatory components. tence of three-frequency quasiperiodicity-appearing, for example, through a secondary Neimark-Sacker bifurcationwas conjectured to be unlikely in real systems for which small inhomogeneities and noise are unavoidable. However, a variety of numerical simulations ${ }^{48,49}$ and some experiments (usually involving periodic forcing, ${ }^{50}$ but see Ref. 51) have shown that stable three-frequency quasiperiodic motion is possible.

In our system, apart from the numerical evidence of three-frequency quasiperiodic behavior (cf. Sec. II C and Ref. 33) there are other arguments that allow us to understand why a $\mathbb{T}^{3}$ can be stable in this system. Namely, Rand ${ }^{52}$ proved that in systems with rotational symmetry there is suppression of lockings in modulated rotating waves. This is because one can make a coordinate transformation to a system with one less rotation, i.e., in the transformed setting the system only has one frequency and exhibits no lockings. In a different context, this result allows to understand the absence of lockings in meandering spiral waves in homogeneous excitable media. ${ }^{53}$ As our system is discrete, this symmetry would be only exact in the continuum limit, but some inhibition of the lockings (usually precursors of the transition to chaos) should be expected. A second factor that is also expected to help in keeping stable the $T^{3}$ attractor in a range is the disparity in the frequencies of the torus, that should narrow the corresponding Arnold tongues, and, thus, make resonant interactions (that destroy the torus) less likely and efficient.

In our experimental setup it is difficult to discern unambiguously that we have observed $T^{3}$ behavior, which is true also to some extent in the above mentioned experimental references, especially because the parameter range in which it is observed in the numerical simulations, cf. Sec. II C, is so narrow, namely $35.09384 \leqslant R \leqslant 35.0955$. Although for this narrow range one should be able to resolve the phenomenon with very fine resolution, and also taking into account the unavoidable sources of noise and tolerances in the system, we present now results that are compatible with the existence of three-frequency quasiperiodicity in our electronic setup. Nonetheless, the narrowness of the range in $R$ found in the numerical study makes the analysis of the electronic setup in terms of three-frequency quasiperiodicity not absolutely univocal. The 3-torus appears as a very slow modulation of the former 2-torus [see Fig. 5(c)]. Looking at the Lissajous figure on the oscilloscope, it looks like a torus whose size oscillates periodically.

The best way to characterize the 3-torus is to compute the fast Fourier transform to identify the third frequency. The problem arising due to the onset of a very small frequency (on the order of $2 \mathrm{~Hz}$ ) is solved using the data acquisition board, that allows us to collect a large number of data.

The result is shown in Fig. 5(b), where $f_{1}$ and $f_{2}$ are the former frequencies and $f_{3}$ is the third (and lowest) frequency. This one is about two orders of magnitude smaller than $f_{2}$, in a similar fashion to the behavior found in some numerical works (in a normal form of two interacting Hopf bifurcations with symmetry ${ }^{54}$ and in a forced Taylor-Couette flow ${ }^{49}$ ).

It is interesting to note the effect of the new very low frequency on the power spectrum. In the 2-torus case, there are linear combinations of the two incommensurate frequencies giving sharp peaks [see Fig. 4(b)]. However, for the 3-torus the existence of an additional frequency much smaller than the other two makes to appear many peaks corresponding to linear combinations of the former peaks with the very low frequency. As a result, this causes the peaks corresponding to frequencies $f_{1}$ and $f_{2}$ (and linear combina- 

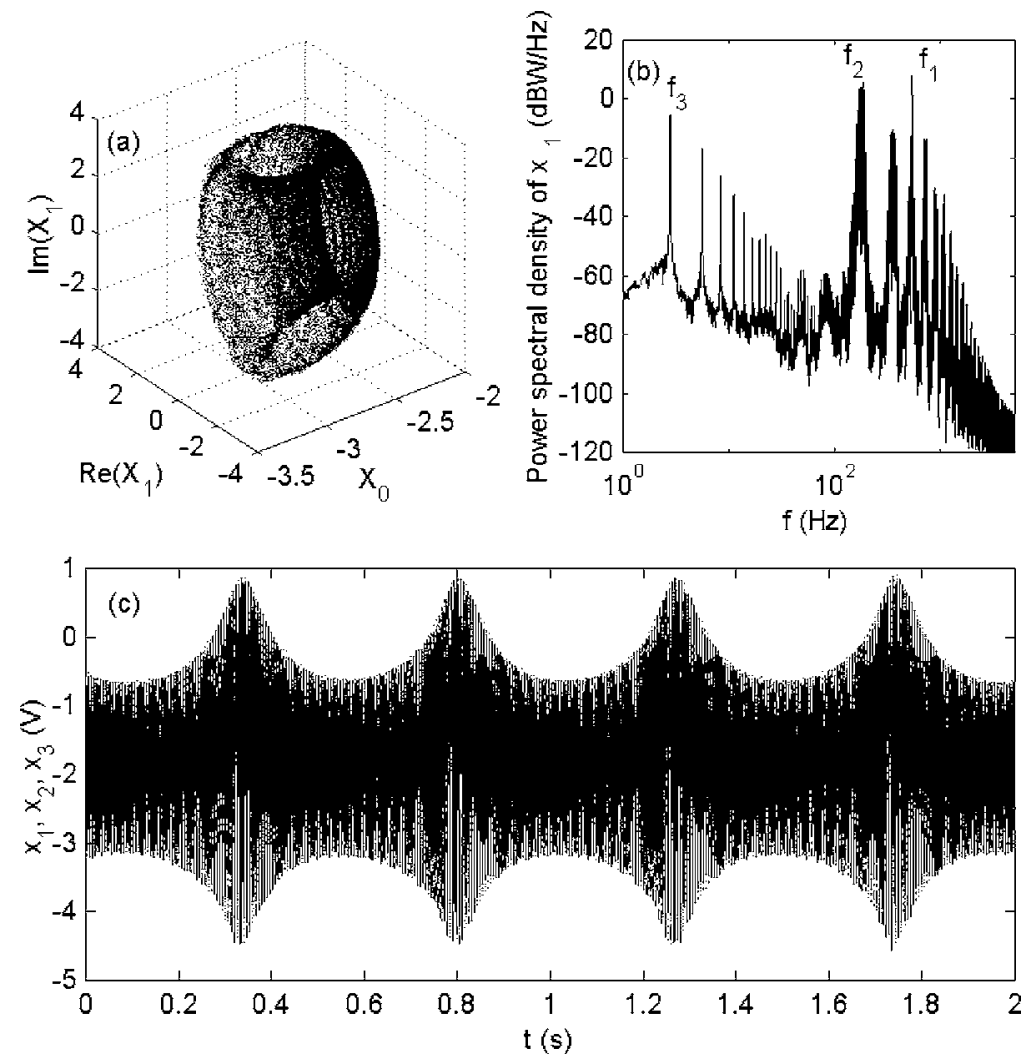

FIG. 5. Three-frequency quasiperiodic motion: experimental results. (a) Poincaré section showing the densely filled surface of a 2-torus. The Poincaré section has been defined by Eq. (6). The axes are built with the $x$ coordinate: uniform $(k=0)$ mode, real and imaginary parts of $k=1$ mode (see the caption of Fig. 3 for an explanation). (b) Power spectrum of variable $\mathrm{x}_{1}(\mathrm{t})$. Besides the two previous incommensurate frequencies, there is an additional third frequency $f_{3} \approx 2 \mathrm{~Hz} \ll f_{1}, f_{2}$. Interaction with this frequency (linear combinations) causes broadening of the peaks of the two pre-existing frequencies. A log-log representation is used to better resolve the peak associated with $f_{3}$, otherwise it would be indistinguishable from the axis of ordinates. (c) Temporal series of variables $\mathrm{x}$ for the three circuits; the third frequency is the envelope that modulates the amplitude of the two other oscillations. Notice the different time scale with respect to Figs. 3(c) and 4(c). tions of them) to broaden; as may be seen in Fig. 5(b) (notice that we are using a logarithmic scale for both axes). It is difficult to get a sharper peak for $f_{3}$, because due to storage limitations in the data acquisition process we could not use more than 176 periods of the low frequency.

We can also face the problem of the geometric visualization of a 3-torus. Note that its dimension is three, the same as our space $\left(\mathbb{R}^{3}\right)$. Also the attractor of the CRW regime has a high fractal dimension. Therefore, to visualize these attractors a Poincaré section is very useful because it reduces the dimension of the attractors in one unity. It is shown in Fig. 5(a) that the result of taking the Poincaré section of the 3-torus, can be recognized as a 2-torus (cf. Fig. 2 of Ref. 33).

\section{Chaotic rotating wave}

This section summarizes the results for the three regions (see Figs. 1 and 2) where the CRW is found. Recall that a CRW is defined as a state where chaotic motion exists at the same time as an oscillation with phase difference of $2 \pi / n$ between adjacent units.

If we start our path at some $R$ with the 3-tori attractors, we find that as long as the parameter $R$ is decreased the three-tori become larger and larger. Then, at some point both mirror tori seem to merge to give rise to the CRW, such that trajectories visit both sides of the phase space. Nevertheless, a careful numerical examination indicates that there exists a very small interval of $R$ where the chaotic and the 3-tori attractors coexist. This is not surprising because, as we mentioned above, a single Lorenz system also exhibits multista- bility in a small interval of $R$. The mechanism creating the (high-dimensional) chaotic attractor is a global bifurcation that was explained in detail in Ref. 55.

In region III the trajectory of each oscillator is similar to that found for the 3-torus, but now, there are jumps from one to other side of phase space. That is, the trajectory seems to jump between the former 3-tori. This can be seen in Fig. 6(c). The Poincaré section is shown in Fig. 6(a) where the two lobes appear in both regions corresponding to the (previous) symmetry-related 3-tori. The attractor looks blurred because the dynamics in each lobe is more complex and also due to the jumps between both lobes. Note that according to the Kaplan-Yorke formula ${ }^{56}$ the chaotic attractor has an information dimension above four (see Fig. 2) and therefore the Poincaré section saturates $\mathbb{R}^{3}$. Also, in Fig. 6(b), as expected for chaos, we observe a wideband power spectrum. The peaks of $f_{1}$ and $f_{2}$ are clearly distinguishable in region III as, at least close to the onset in which the highdimensional attractor appears, chaos is associated to jumps between the two lobes, and the typical frequency of these jumps is quite low, of the order of $f_{3}$ [cf. Fig. 6(c)].

Region I, at the left most part of the interval of $R$ where the CRW is found, shows a dynamics previously reported in Ref. 19. The motion is apparently very simple because the trajectory of the average of the three oscillators (mode $k=0$ ) is very similar to that of an isolated Lorenz oscillator [see Fig. 7(c)]. Superimposed to that chaotic motion, every circuit exhibits an oscillating component with a phase difference of $2 \pi / 3$ between consecutive oscillators. ${ }^{58}$

Region II is clearly hyperchaotic [inset of Fig. 2(a) shows an extra slightly positive LE]. The dynamics exhibits 

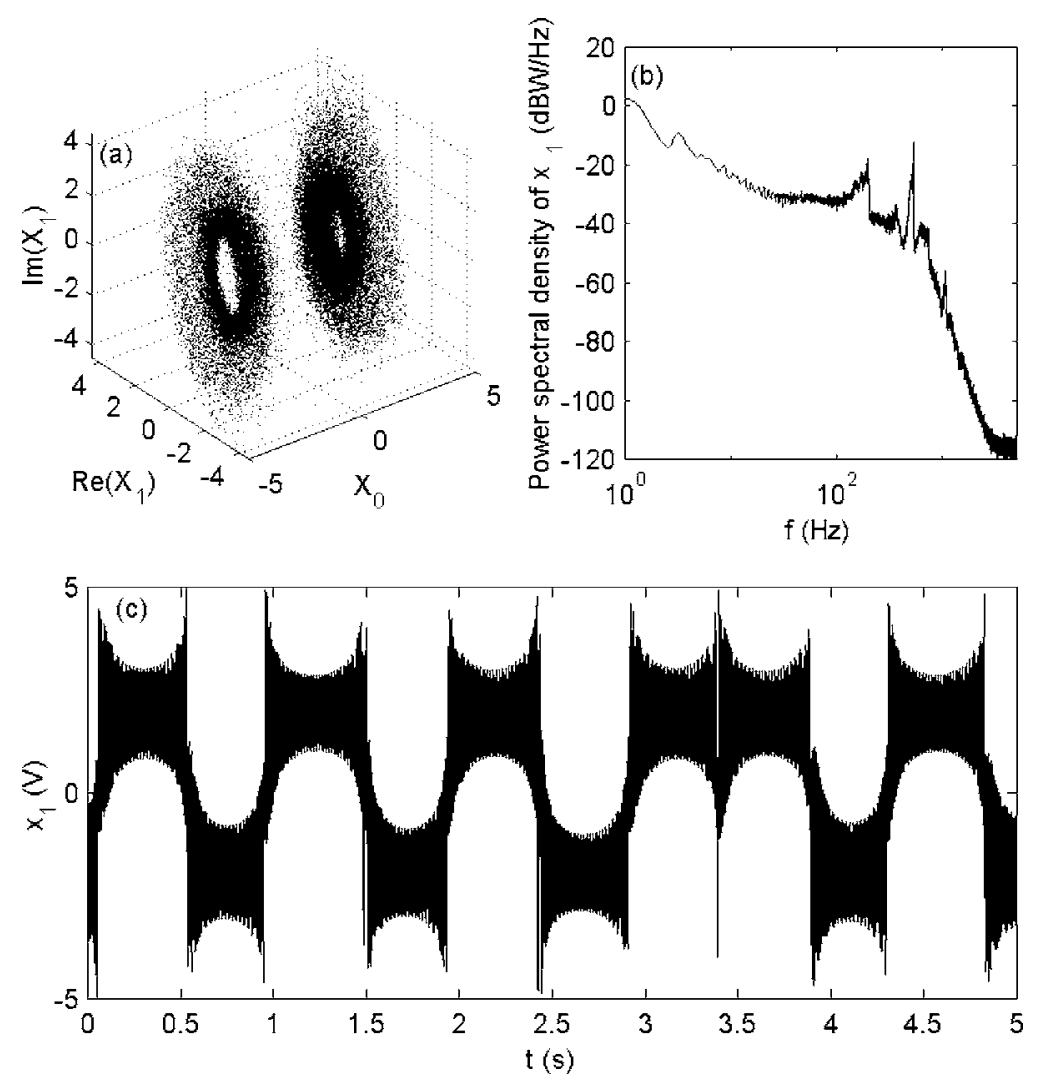

FIG. 6. Chaotic rotating wave, region III: experimental results. (a) Poincaré section for the system with the attractor compound of the two lobes (see Figs. 3 and 5 or an explanation of the coordinates). The attractor is blurred because of the complexity of the new state compared to the former 3-tori. The Poincaré section has been defined by Eq. (6). (b) Power spectrum of variable $\mathrm{x}_{1}(\mathrm{t})$. There are still two peaks at the frequencies $f_{1}$ and $f_{2}$. (c) Temporal series of variable $\mathrm{x}$ for one circuit showing the jumps between the two lobes. some kind of intermittency between the behaviors observed in regions I and III. One may speculate the existence of an interior crisis in the attractor. It may be seen in Fig. 7(b) that the long intervals in one of the sides of the attractor (like in region III) are interrupted by intervals where both sides are visited in a comparably faster way (like in region I).

\section{CONCLUSIONS}

In the present work we present a joint experimental and numerical study of the route that connects some patterns occurring in a ring of three coupled Lorenz oscillators, involving, namely, periodic and chaotic rotating discrete rotating waves, and also synchronized chaos. The occurrence of the different behaviors has been first characterized in parameter space by analyzing the largest Lyapunov exponents. These behaviors have been then identified by direct analysis of the experiment, with the aid of power spectrum and in more detail through the use of Poincaré sections.

A brief synopsis of the observed behaviors in a line in parameter space (keeping $\sigma$ and $b$ constant, and increasing $R)$ is as follows. Complete synchronization among the cells of the ring becomes unstable through a Hopf instability that is characterized by a new fast frequency that induces a phase shift of $2 \pi / n$ between adjacent units. It is to be stressed that just above the loss of synchronization (region I) two Lyapunov exponents vanish (at least at our precision level), and only when $R$ is increased further hyperchaos is found (region II). The hyperchaotic regime exhibits an intermittent behavior between characteristic dynamics of regions I and III. Curiously enough, region III (existing at larger values of $R$ ) exhibits, again, two vanishing LEs. Finally, chaos disap-
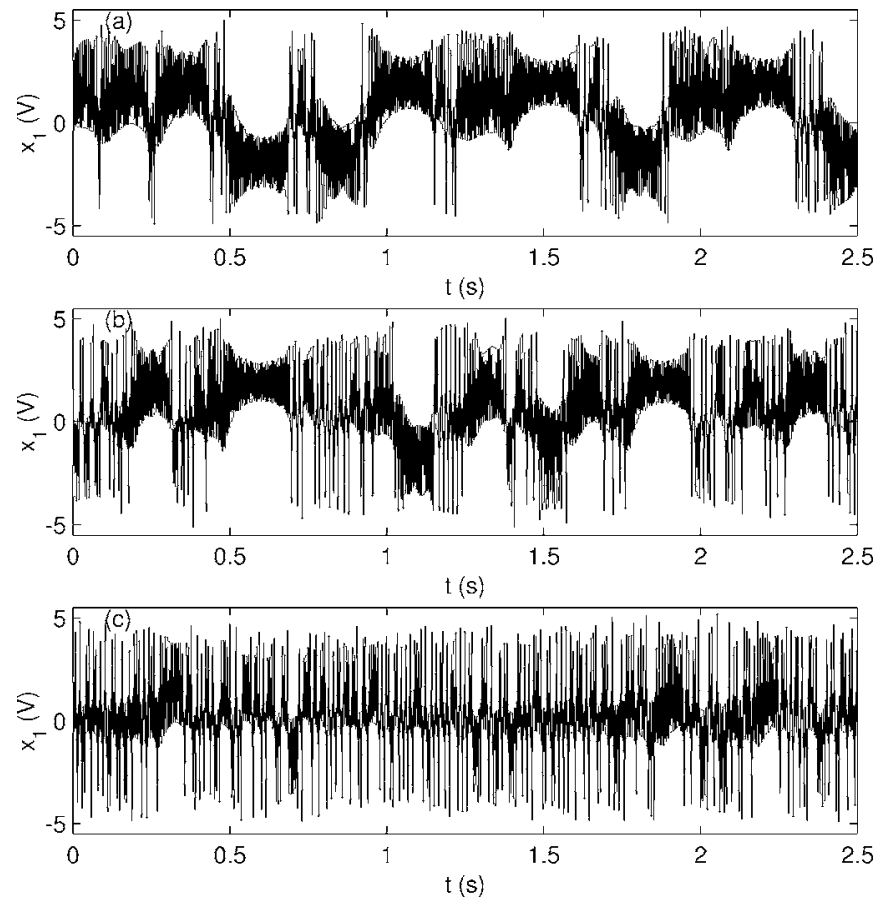

FIG. 7. Chaotic rotating waves: experimental results. As the parameter $R$ is lowered the system passes from region III to regions II and I, as was explained before (see Figs. 1 and 2). (a) Time series for a value of $R$ slightly smaller than in Fig. 6(c). It corresponds to approximately the transition point between regions III and II. (b) Region II. The motion is intermittent, alternating dynamics between characteristic motions of regions I and III. This results in two positive Lyapunov exponents. (c) Region I. Now, there is a chaotic motion-with frequent jumps between both lobes-similar to the Lorenz attractor (with a superimposed oscillation that is not visible in this time scale). 
pears giving rise to three-frequency quasiperiodicity. Later on, two consecutive (inverse) Neimark-Sacker bifurcations give rise to a very coherent (symmetric under $\mathbf{Z}_{3} \times \mathbf{S}^{1}$ ) state: a periodic rotating wave. Therefore we have shown here a route between two states (complete chaotic synchronization and periodic rotating wave) that have been studied in different contexts, as we explained in the Introduction.

The close match between the numerical predictions and the experimental results clearly indicates that the results appear to be robust under the presence of the unavoidable noise present in a physical experiment. This is true even when the system is highly nonhyperbolic, e.g., in the parameter regions in which one positive and two zero Lyapunov exponents are present, the attractor contains an infinite set of unstable two-dimensional tori. This close match between theory and experiment is also reassuring taking into account that potentially the system is also a candidate to being subject to obstructions to deterministic modeling, ${ }^{30}$ as another possible manifestation of nonhyperbolicity.

\section{ACKNOWLEDGMENTS}

We acknowledge financial support from MEC (Spain) and FEDER through Grant Nos. BFM2001-0341-C02-02, FIS2004-00953 (CONOCE2), and FIS2004-05073-C04-03, and from the Fundación Memoria de Samuel Solórzano Barruso.

${ }^{1}$ T. Bohr, M. H. Jensen, G. Paladin, and A. Vulpiani, Dynamical Systems Approach to Turbulence (Cambridge University Press, Cambridge, 1998). ${ }^{2}$ E. N. Lorenz, J. Atmos. Sci. 20, 130 (1963).

${ }^{3}$ C. Huygens, Oeuvres complétes de Christiaan Huygens (Martinus Nijhoff, The Hague, 1888-1850), Vol. 5, pp. 241-262.

${ }^{4}$ I. Blekhman, Synchronization in Science and Technology (Nauka, Moskow, 1981).

${ }^{5}$ H. Fujisaka and T. Yamada, Prog. Theor. Phys. 69, 32 (1983); A. S. Pikovsky, Z. Phys. B: Condens. Matter 55, 149 (1984); V. S. Afraimovich, N. N. Verichev, and M. I. Rabinovich, Radiophys. Quantum Electron. 29, 795 (1986)

${ }^{6}$ L. M. Pecora and T. L. Carroll, Phys. Rev. Lett. 64, 821 (1990).

${ }^{7}$ A. Pikovsky, M. Rosenblum, and J. Kurths, Synchronization: A Universal Concept in Nonlinear Sciences (Cambridge University Press, Cambridge, 2001).

${ }^{8}$ L. M. Pecora, T. L. Carroll, G. A. Johnson, D. J. Mar, and J. F. Heagy, Chaos 7, 520 (1997).

${ }^{9}$ S. Boccaletti, J. Kurths, G. Osipov, D. Valladares, and C. Zhou, Phys. Rep. 366, 1 (2002).

${ }^{10}$ L. M. Pecora, Phys. Rev. E 58, 347 (1998).

${ }^{11}$ A Chaotic Hierarchy, edited by G. Baier and M. Klein (World Scientific, Singapore, 1991)

${ }^{12}$ E. Rodriguez, N. George, J. P. Lachaux, J. Martinerie, B. Renault, and F. Varela, Nature (London) 397, 430 (1999).

${ }^{13}$ M. Golubitsky, I. Stewart, P.-L. Buono, and J. J. Collins, Nature (London) 401, 693 (1999).

${ }^{14}$ J. J. Collins and I. N. Stewart, J. Nonlinear Sci. 3, 349 (1993); Biol. Cybern. 71, 95 (1994).

${ }^{15}$ G. B. Ermentrout, J. Math. Biol. 23, 55 (1985).

${ }^{16}$ P Ashwin, G. P. King, and J. W. Swift, Nonlinearity 3, 585 (1990).

${ }^{17}$ D. G. Aronson, M. Golubitsky, and J. Mallet-Paret, Nonlinearity 4, 903 (1991).

${ }^{18}$ M. A. Matías, V. Pérez-Muñuzuri, I. P. Mariño, M. N. Lorenzo, and V. Pérez-Villar, Phys. Rev. Lett. 78, 219 (1997); M. A. Matías and J. Güémez, ibid. 81, 4124 (1998).

${ }^{19}$ E. Sánchez and M. A. Matías, Int. J. Bifurcation Chaos Appl. Sci. Eng. 9, 2335 (1999)

${ }^{20}$ G. Hu, Y. Zhang, H. A. Cerdeira, and S. Chen, Phys. Rev. Lett. 85, 3377 (2000); Y. Zhang, G. Hu, and H. A. Cerdeira, Phys. Rev. E 64, 037203 (2001).
${ }^{21}$ E. Sánchez and M. A. Matías, Phys. Rev. E 57, 6184 (1998).

${ }^{22}$ I. P. Mariño, V. Pérez-Muñuzuri, and M. A. Matías, Int. J. Bifurcation Chaos Appl. Sci. Eng. 8, 1733 (1998).

${ }^{23}$ M. A. Matías, J. Güémez, V. Pérez-Muñuzuri, I. P. Mariño, M. N. Lorenzo, and V. Pérez-Villar, Europhys. Lett. 37, 379 (1997); E. Sánchez, M. A. Matías, and V. Pérez-Muñuzuri, IEEE Trans. Circuits Syst., I: Fundam. Theory Appl. 47, 644 (2000).

${ }^{24}$ G. Hu, J. Z. Yang, W. Q. Ma, and J. H. Xiao, Phys. Rev. Lett. 81, 5314 (1998); G. Hu, J. Z. Yang, and W. Liu, Phys. Rev. E 58, 4440 (1998).

${ }^{25}$ H. L. Yang and A. S. Pikovsky, Phys. Rev. E 60, 5474 (1999).

${ }^{26}$ J. Yang, Phys. Rev. E 61, 6521 (2000).

${ }^{27}$ M. Zhang, G. Hu, and J. Yang, Phys. Rev. E 62, 2963 (2000).

${ }^{28}$ E. Mosekilde, Topics in Nonlinear Dynamics (World Scientific, Singapore, 1996).

${ }^{29}$ D. G. Luchinsky, P. V. E. McClintock, and M. I. Dykman, Rep. Prog. Phys. 61, 889 (1998).

${ }^{30}$ Y.-C. Lai, C. Grebogi, and J. Kurths, Phys. Rev. E 59, 2907 (1999); Y.-C. Lai, D. Lerner, K. Williams, and C. Grebogi, ibid. 60, 5445 (1999); Y.-C. Lai and C. Grebogi, Phys. Rev. Lett. 82, 4803 (1999).

${ }^{31}$ L. Jaeger and H. Kantz, Physica D 105, 79 (1997).

${ }^{32}$ J. Güémez and M. A. Matías, Phys. Rev. E 52, R2145 (1995).

${ }^{33}$ D. Pazó, E. Sánchez, and M. A. Matías, Int. J. Bifurcation Chaos Appl. Sci. Eng. 11, 2683 (2001).

${ }^{34}$ J. F. Heagy, T. L. Carroll, and L. M. Pecora, Phys. Rev. E 50, 1874 (1994).

${ }^{35}$ Nevertheless, for chaotic attractors, transversal instabilities of some unstable periodic orbits may induce intermittent losses of synchronization; this is known as attractor bubbling.

${ }^{36}$ G. Benettin, L. Galgani, A. Giorgilli, and J. M. Strelcyn, Meccanica 15, 9 (1980).

${ }^{37}$ I. Shimada and T. Nagashima, Prog. Theor. Phys. 61, 1605 (1979).

${ }^{38}$ A. Wolf, J. B. Swift, H. L. Swinney, and J. A. Vastano, Physica D 16, 285 (1985).

${ }^{39}$ W. H. Press, S. A. Teukolsky, W. T. Vetterling, and B. P. Flannery, $N u-$ merical Recipes in Fortran 77: The Art of Scientific Computing, 2nd ed. (Cambridge University Press, Cambridge, 1992).

${ }^{40}$ E. Ott and J. C. Sommerer, Phys. Lett. A 188, 39 (1994).

${ }^{41}$ P. Ashwin, J. Buescu, and I. Stewart, Phys. Lett. A 193, 126 (1994).

${ }^{42}$ J. A. Yorke and E. D. Yorke, J. Stat. Phys. 21, 263 (1979).

${ }^{43}$ Z. Liu, Y.-C. Lai, and M. A. Matías, Phys. Rev. E 67, 045203(R) (2003).

${ }^{44}$ A. Pujol-Peré, O. Calvo, M. A. Matías, and J. Kurths, Chaos 13, 319 (2003).

${ }^{45}$ D. Pazó, I. P. Mariño, V. Pérez-Villar, and V. Pérez-Muñuzuri, Int. J. Bifurcation Chaos Appl. Sci. Eng. 10, 2533 (2000).

${ }^{46}$ D. Pazó, Ph.D. thesis, Universidade de Santiago de Compostela (2003).

${ }^{47}$ S. Newhouse, D. Ruelle, and F. Takens, Commun. Math. Phys. 64, 35 (1978).

${ }^{48}$ C. Grebogi, E. Ott, and J. A. Yorke, Phys. Rev. Lett. 51, 339 (1983); Physica D 15, 354 (1985); P. Battelino, Phys. Rev. A 38, 1495 (1988); U. Feudel, W. Jansen, and J. Kurths, Int. J. Bifurcation Chaos Appl. Sci. Eng. 3, 131 (1993); V. Anishchenko, M. Safonova, U. Feudel, and J. Kurths, ibid. 4, 595 (1994); U. Feudel, M. Safonova, J. Kurths, and V. Anishchenko, ibid. 6, 1319 (1996)

${ }^{49}$ J. M. Lopez and F. Marques, Phys. Rev. Lett. 85, 972 (2000).

${ }^{50}$ J. P. Gollub and S. V. Benson, J. Fluid Mech. 100, 449 (1980); A. Cumming and P. S. Linsay, Phys. Rev. Lett. 60, 2719 (1988); T. Yazaki, Phys. Rev. E 48, 1806 (1993); R. Alaggio and G. Rega, Physica D 137, 70 (2000).

${ }^{51}$ T. Schneider, H. Heng, and W. Martienssen, Europhys. Lett. 22, 499 (1993).

${ }^{52}$ D. Rand, Arch. Ration. Mech. Anal. 79, 1 (1982).

${ }^{53}$ D. Barkley, in Chemical Waves, edited by R. Kapral and K. Showalter (Kluwer, Dordrecht, 1995), p. 163.

${ }^{54}$ X.-J. Wang and G. Nicolis, Physica D 26, 140 (1987).

${ }^{55}$ D. Pazó and M. A. Matías, Europhys. Lett. 72, 176 (2005).

${ }^{56}$ According to the Kaplan-Yorke conjecture, Ref. 57 says that the information dimension is $D_{1}=K+\sum_{j=1}^{K}\left(\lambda_{j} /\left|\lambda_{K+1}\right|\right)$, being $K$ the largest integer such that $\sum_{j=1}^{K} \lambda_{j} \geqslant 0$, where the $\lambda_{j}$ are the Lyapunov exponents ordered from larger to smaller.

${ }^{57}$ J. L. Kaplan and J. A. Yorke, in Functional Differential Equations and Approximation of Fixed Points, edited by H. O. Walther and H. O. Peitgen (Springer, Berlin, 1979), p. 204.

${ }^{58}$ E. Sánchez, Ph.D. thesis, Universidad de Salamanca (2000). 